\title{
Rat engineered heart tissue: a novel tool in the safety pharmacology toolkit?
}

\author{
Jordi Heijman • Dobromir Dobrev
}

Received: 29 August 2014/Accepted: 29 August 2014/Published online: 11 September 2014

(C) Springer-Verlag Berlin Heidelberg 2014

Adverse drug effects are a major problem in clinical practice and are responsible for more than $5 \%$ of hospital admissions [16]. Drug-induced proarrhythmia is among the most severe adverse side effects with potentially fatal consequences. Over the last decades, several drugs have been taken off the market, or have been restricted in their application, due to concerns about proarrhythmic side effects [10]. Accordingly, cardiac safety testing is now mandatory before a new compound can be approved for clinical use. Many proarrhythmic compounds have been found to inhibit the rapid delayed-rectifier $\mathrm{K}^{+}$-current $\left(I_{\mathrm{Kr}}\right)$ by blocking the underlying channel encoded by the human ether-a-go-go-related gene $(h E R G)$, resulting in excessive prolongation of cardiac repolarization, which has been associated with drug-induced "Torsade-de-Pointes" (TdP) arrhythmias. Consequently, current cardiac safety assays mainly involve in vitro screening of $I_{\mathrm{Kr}}$ inhibition (using drug-binding assays, fluorescent thallium flux assays, or automated patch-clamp), followed by in vivo analyses of QT-interval prolongation in large animal models and a "thorough QT study" in humans (reviewed in detail in [6, $8,12,17])$. Although screening for $I_{\mathrm{Kr}}$ inhibition can be performed easily in high-throughput systems, it is now well accepted that evaluating $I_{\mathrm{Kr}}$ inhibition in non-cardiomyocytes only is insufficient to accurately predict the torsadogenic potential of novel compounds $[6,8]$. In vivo studies in large animal models, on the other hand, show good (but

This comment refers to the article available at doi:10.1007/s00395014-0436-7.

J. Heijman · D. Dobrev $(\varangle)$

Institute of Pharmacology, Faculty of Medicine, University

Duisburg-Essen, Hufelandstr 55, 45122 Essen, Germany

e-mail: dobromir.dobrev@uk-essen.de not perfect) correlation with arrhythmogenic risk in patients, but their high costs, low throughput, and ethical concerns preclude their application for all but the most promising candidate compounds. It is unknown how many safe compounds with potential beneficial clinical use have been eliminated due to $I_{\mathrm{Kr}}$ inhibition as a result of the present 'fail early, fail cheaply' approach.

Ectopic activity and reentry are well-established mechanisms for the initiation and maintenance of both atrial and ventricular arrhythmias. Although arrhythmias are intrinsically multicellular phenomena, and this aspect should be taken into account during cardiac safety testing, basic research has provided a wealth of information about the underlying molecular and cellular mechanisms promoting arrhythmias [5, 7, 14, 21]. Cardiac electrophysiology is controlled by a large number of ion channels and transporters, each of which is modulated by several signaling pathways. This system allows for various feed-back mechanisms and ensures that there is not a single point of failure. Accordingly, an acute insult to a single component is rarely sufficient to initiate and maintain an arrhythmia. Indeed, even in a monogenic disease in a large founder population with a dominant-negative mutation in the $K C N Q 1$ gene, resulting in a pronounced reduction in the slow delayed-rectifier $\mathrm{K}^{+}$-current, the clinical phenotype was extremely diverse [1]. Thus, other risk factors including a genetic predisposition, disease-related remodeling, neurohumoral factors, and drug effects can importantly influence the development and maintenance of cardiac arrhythmias [5, 7, 18]. This complexity also highlights the urgent need for novel integrative cardiac safety assays that can bridge the gap between available in vitro and in vivo systems and can provide a reliable assessment of potential proarrhythmic consequences of novel pharmacological compounds. 
In the present issue of Basic Research in Cardiology, Eder et al. [4] present a novel experimental system with potential for safety pharmacology. In rat engineered heart tissue (EHT), drug effects were quantified non-invasively by alterations in contractility. Since contractile parameters of spontaneously beating EHTs can be determined automatically using a video camera while samples are maintained under relatively physiological conditions $\left(37^{\circ} \mathrm{C}\right.$, $7 \% \mathrm{CO}_{2}$, and in the presence of $50 \mathrm{nmol} / \mathrm{L}$ epinephrine to simulate basal autonomic tone) in 24-well plates, this system provides a multicellular, medium-throughput option for safety pharmacology.

Eder et al. [4] investigated the relation between contractile parameters and electrical properties in EHTs using pharmacological compounds with well-characterized electrophysiological effects. As previously shown in isolated rat cardiomyocytes, inhibition of the transient-outward $\mathrm{K}^{+}$-current $\left(I_{\mathrm{to}}\right)$ prolonged action potential duration (APD), whereas epinephrine shortened APD in EHTs. Changes in APD induced by 4-AP or epinephrine correlated well with changes in $\mathrm{Ca}^{2+}$-transient decay and relaxation time (T2; measured from peak contraction to $20 \%$ relaxation). On the other hand, inhibition of $I_{\mathrm{Kr}}$ (with E-4031) or $I_{\mathrm{Ks}}$ (with HMR1556) did not affect T2 duration in EHTs, in agreement with the unaltered APD reported in other studies [22]. In contrast, combined inhibition of $I_{\mathrm{Kr}}$ and $I_{\mathrm{Ks}}$ did produce a significant prolongation in relaxation time in rat EHTs. These data suggest that these currents may contribute to the repolarization reserve of rat EHTs. Nonetheless, the link between increased relaxation time and repolarization prolongation should be evaluated extremely cautiously, since relaxation time is also modulated by changes in myofilament properties. Compounds such as levosimendan, a positive inotropic substance that sensitizes myofilament $\mathrm{Ca}^{2+}$ binding [15], would be expected to prolong relaxation time, even though it has been shown to shorten APD by opening ATP-dependent $\mathrm{K}^{+}$-channels [23]. Similarly, previous work by the authors of the present study indicated that mutations in the ankyrin repeat domain 1 (ANKRDl) gene associated with hypertrophic cardiomyopathy can affect contraction parameters of rat EHTs [2], and that EHTs from knock-in mice with a myosin-binding protein $\mathrm{C}$ binding show altered druginduced contractile parameters, but normal $\mathrm{Ca}^{2+}$-transient properties [20]. In addition, contractile properties are strongly modulated by the frequency of activation. Since the EHTs in the study by Eder et al. were not paced, activation rates were quite irregular, which may further complicate the use of $\mathrm{T} 2$ as a surrogate for repolarization duration.

On the other hand, Eder et al. [4] also show that inhibition of the $\mathrm{Na}^{+} / \mathrm{Ca}^{2+}$ exchanger with SEA0400 or inhibition of cardiac ryanodine receptor channels with JTV519 prevented spontaneous contractions in the presence of 4-AP. These findings suggest that, in contrast to many other in vitro safety assays, EHTs might be useful to assess ectopic activity due to $\mathrm{Ca}^{2+}$-handling abnormalities. Spontaneous sarcoplasmic reticulum (SR) $\mathrm{Ca}^{2+}$-release events have been shown to play a major proarrhythmic role in various pathophysiological conditions, including heart failure and atrial fibrillation [7, 13, 14], and the ability to detect these proarrhythmic events is a highly desirable property for novel safety assays. However, since there are important differences in $\mathrm{Ca}^{2+}$-handling properties between rodents and humans, as well as between different regions of the heart (e.g., atria versus ventricles [3]), and different developmental stages, it remains uncertain whether druginduced $\mathrm{Ca}^{2+}$-handling abnormalities in rat EHTs accurately reflect proarrhythmic risk in patients. Furthermore, spontaneous SR $\mathrm{Ca}^{2+}$-release events may themselves also promote APD prolongation by reducing $\mathrm{Ca}^{2+}$-dependent inactivation of the L-type $\mathrm{Ca}^{2+}$-channel during the subsequent AP [9]. This phenomenon could potentially explain the increase in $\mathrm{T} 2$ duration observed in rat EHTs following application of thapsigargin [4] (which is expected to lower SR $\mathrm{Ca}^{2+}$ load and decrease inactivation of L-type $\mathrm{Ca}^{2+}$ channels), and further highlights the complexity of the interactions among repolarization, $\mathrm{Ca}^{2+}$ handling, and contraction.

To assess the potential of rat EHTs for cardiac safety testing, Eder et al. [4] evaluated the proarrhythmic effects of various clinically relevant compounds. The authors should be commended for the large number of compounds that were tested. In addition to 28 new chemical entities, 49 compounds with varying degrees of proarrhythmic potential were investigated. Given the lack of sensitivity to inhibition of two main repolarizing currents $\left(I_{\mathrm{Kr}}\right.$ and $\left.I_{\mathrm{Ks}}\right)$, which have been shown to at least contribute to the proarrhythmic potential of drugs in patients, it is not surprising that $\mathrm{T} 2$ prolongation in rat EHT was unable to clearly discriminate between compounds with a low or high risk of drug-induced proarrhythmia. Despite this lack of specificity in identifying potentially proarrhythmic compounds based on T2 prolongation with rat EHTs, the present work shows that EHTs allow a characterization of a relatively large number of compounds. In addition to the non-invasive characterization of $\mathrm{Ca}^{2+}$ handling, making them suitable for medium-throughput studies, EHTs have several other desirable properties for a cardiac safety assay positioned between current in vitro and in vivo options (Fig. 1). For example, EHTs allow analysis of both acute and long-term/chronic applications of a compound. Recent work by Yang et al. [22] has shown that several substances that were considered specific $I_{\mathrm{Kr}}$ blockers can additionally cause an increase in late $\mathrm{Na}^{+}$-current $\left(I_{\mathrm{NaL}}\right)$ when applied 


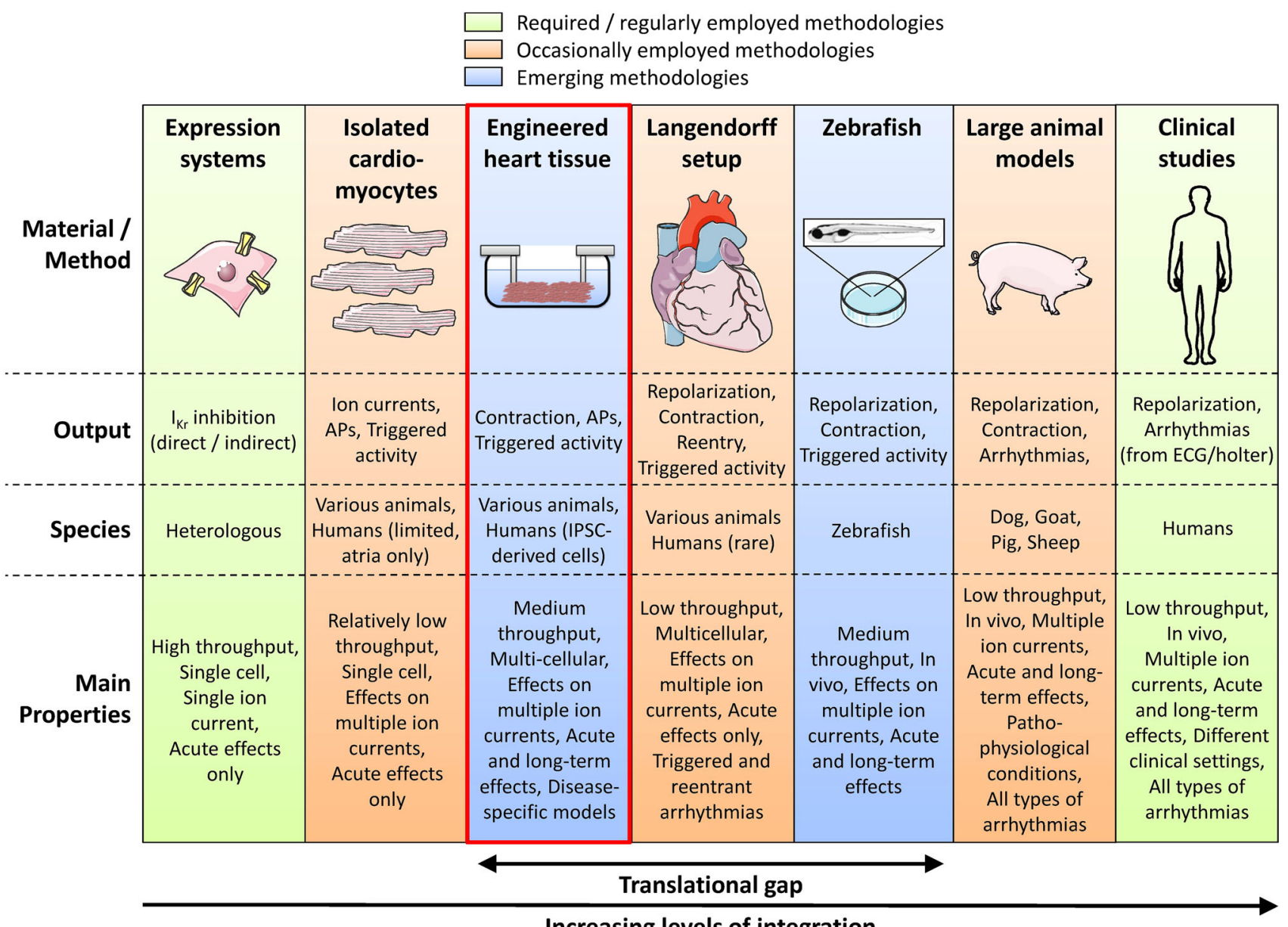

Increasing levels of integration

Fig. 1 Methodologies used for cardiac safety testing. Analysis of $I_{\mathrm{Kr}}$ inhibition in expression systems and QT-interval analysis in vivo represent those methodologies on opposite ends of the spectrum of integration that are currently required in cardiac safety testing (green). Other methods including isolated cardiomyocytes and

chronically and that this may contribute to their torsadogenic potential. EHTs would likely be able to identify such effects.

Finally, there are several potential extensions of the present work that would help to address some of the limitations. The use of EHTs based on human-induced pluripotent stem cell (IPSC) or embryonic stem cell (ES)-derived cardiac tissue could help to overcome some of the speciesdependent differences, and is expected to provide a better indication of the torsadogenic potential of a compound in patients. A proof-of-principle using EHTs from human ES and few selected pharmacologic compounds has already been published [19]. Although stem cell-derived cardiomyocytes have a relatively immature phenotype and the application of EHT from such cells for the screening of numerous compounds is at present still too costly, further developments in this field are expected to result in a humanspecific system that can be employed for medium-
Langedorff-perfused hearts are also occasionally used (orange). Newer methodologies (blue), including the engineered heart tissue (EHT) described by Eder et al. [4] (indicated by a red border), attempt to bridge the translational gap between recording of a single ion current and in vivo recordings

throughput studies [19]. Nonetheless, further validation of relaxation time as a surrogate for repolarization durations in human IPSC-derived EHTs is also needed. Human EHTs could be characterized in their native rhythm as well as under paced conditions to remove some of the confounding effects of irregular rates. Moreover, the libraries of IPS cells for various diseases that are currently being built by various institutions (reviewed in [11]) could provide an opportunity to perform safety assays in EHTs incorporating specific risk factors known to promote the incidence of drug-induced proarrhythmia, thereby providing a better risk assessment for the patients that will actually receive such drugs.

In summary, the study by Eder et al. [4] highlights that EHTs might be a new tool in the cardiac safety pharmacology toolkit, combining reasonable throughput due to non-invasive assessment with the ability to determine $\mathrm{Ca}^{2+}$-handling abnormalities. Further research on human IPSC-derived EHTs may provide the human-specific 
context needed to overcome some of the limitations identified in rat EHTs and result in a novel integrative system that can bridge the gap between existing cardiac safety assays.

Acknowledgments The authors' work is supported by the European Network for Translational Research in Atrial Fibrillation (EUTRAF: 261057), the German Federal Ministry of Education and Research through DZHK (German Center for Cardiovascular Research), and the Deutsche Forschungsgemeinschaft (Do 769/1-3).

\section{References}

1. Brink PA, Crotti L, Corfield V, Goosen A, Durrheim G, Hedley P, Heradien M, Geldenhuys G, Vanoli E, Bacchini S, Spazzolini C, Lundquist AL, Roden DM, George AL Jr, Schwartz PJ (2005) Phenotypic variability and unusual clinical severity of congenital long-QT syndrome in a founder population. Circulation 112:2602-2610. doi:10.1161/CIRCULATIONAHA.105.572453

2. Crocini C, Arimura T, Reischmann S, Eder A, Braren I, Hansen A, Eschenhagen T, Kimura A, Carrier L (2013) Impact of ANKRD1 mutations associated with hypertrophic cardiomyopathy on contraction parameters of engineered heart tissue. Basic Res Cardiol 108:349. doi:10.1007/s00395-013-0349-x

3. Dobrev D, Teos LY, Lederer WJ (2009) Unique atrial myocyte $\mathrm{Ca}^{2+}$ signaling. J Mol Cell Cardiol 46:448-451. doi:10.1016/j. yjmcc.2008.12.004

4. Eder A, Hansen A, Uebeler J, Schulze T, Neuber C, Schaaf S, Yuan L, Christ T, Vos MA, Eschenhagen T (2014) Effects of proaarhythmic drugs on relaxation time and beating pattern in rat engineered heart tissue. Basic Res Cardiol (this issue)

5. Grace AA, Roden DM (2012) Systems biology and cardiac arrhythmias. Lancet 380:1498-1508. doi:10.1016/S01406736(12)61462-7

6. Heijman J, Voigt N, Carlsson LG, Dobrev D (2014) Cardiac safety assays. Curr Opin Pharmacol 15:16-21. doi:10.1016/j. coph.2013.11.004

7. Heijman J, Voigt N, Nattel S, Dobrev D (2014) Cellular and molecular electrophysiology of atrial fibrillation initiation, maintenance, and progression. Circ Res 114:1483-1499. doi:10. 1161/CIRCRESAHA.114.302226

8. Hoffmann P, Warner B (2006) Are hERG channel inhibition and QT interval prolongation all there is in drug-induced torsadogenesis? A review of emerging trends. J Pharmacol Toxicol Methods 53:87-105. doi:10.1016/j.vascn.2005.07.003

9. Johnson DM, Heijman J, Bode EF, Greensmith DJ, van der Linde H, Abi-Gerges N, Eisner DA, Trafford AW, Volders PG (2013) Diastolic spontaneous calcium release from the sarcoplasmic reticulum increases beat-to-beat variability of repolarization in canine ventricular myocytes after beta-adrenergic stimulation. Circ Res 112:246-256. doi:10.1161/CIRCRESAHA.112.275735

10. Laverty H, Benson C, Cartwright E, Cross M, Garland C, Hammond T, Holloway C, McMahon N, Milligan J, Park B, Pirmohamed M, Pollard C, Radford J, Roome N, Sager P, Singh S, Suter T, Suter W, Trafford A, Volders P, Wallis R, Weaver R, York M, Valentin J (2011) How can we improve our understanding of cardiovascular safety liabilities to develop safer medicines? Br J Pharmacol 163:675-693. doi:10.1111/j.14765381.2011.01255.x

11. McKernan R, Watt FM (2013) What is the point of large-scale collections of human induced pluripotent stem cells? Nat Biotechnol 31:875-877. doi:10.1038/nbt.2710

12. Meyer T, Sartipy P, Blind F, Leisgen C, Guenther E (2007) New cell models and assays in cardiac safety profiling. Expert Opin Drug Metab Toxicol 3:507-517. doi:10.1517/17425225.3.4.507

13. Nattel S, Dobrev D (2012) The multidimensional role of calcium in atrial fibrillation pathophysiology: mechanistic insights and therapeutic opportunities. Eur Heart J 33:1870-1877. doi:10. 1093/eurheartj/ehs079

14. Neef S, Maier LS (2013) Novel aspects of excitation-contraction coupling in heart failure. Basic Res Cardiol 108:360. doi:10. 1007/s00395-013-0360-2

15. Papp Z, Edes I, Fruhwald S, De Hert SG, Salmenpera M, Leppikangas H, Mebazaa A, Landoni G, Grossini E, Caimmi P, Morelli A, Guarracino F, Schwinger RH, Meyer S, Algotsson L, Wikstrom BG, Jorgensen K, Filippatos G, Parissis JT, Gonzalez MJ, Parkhomenko A, Yilmaz MB, Kivikko M, Pollesello P, Follath F (2012) Levosimendan: molecular mechanisms and clinical implications: consensus of experts on the mechanisms of action of levosimendan. Int J Cardiol 159:82-87. doi:10.1016/j. ijcard.2011.07.022

16. Pirmohamed M, James S, Meakin S, Green C, Scott AK, Walley TJ, Farrar K, Park BK, Breckenridge AM (2004) Adverse drug reactions as cause of admission to hospital: prospective analysis of 18820 patients. BMJ 329:15-19. doi:10.1136/bmj.329.7456. 15

17. Rampe D, Brown AM (2013) A history of the role of the hERG channel in cardiac risk assessment. J Pharmacol Toxicol Methods 68:13-22. doi:10.1016/j.vascn.2013.03.005

18. Sauer AJ, Newton-Cheh C (2012) Clinical and genetic determinants of torsade de pointes risk. Circulation 125:1684-1694. doi:10.1161/CIRCULATIONAHA.111.080887

19. Schaaf S, Shibamiya A, Mewe M, Eder A, Stohr A, Hirt MN, Rau T, Zimmermann WH, Conradi L, Eschenhagen T, Hansen A (2011) Human engineered heart tissue as a versatile tool in basic research and preclinical toxicology. PLoS One 6:e26397. doi:10. 1371/journal.pone.0026397

20. Stöhr A, Friedrich FW, Flenner F, Geertz B, Eder A, Schaaf S, Hirt MN, Uebeler J, Schlossarek S, Carrier L, Hansen A, Eschenhagen T (2013) Contractile abnormalities and altered drug response in engineered heart tissue from Mybpc3-targeted knockin mice. J Mol Cell Cardiol 63:189-198. doi:10.1016/j.yjmcc. 2013.07.011

21. Tsuji Y, Heijman J, Nattel S, Dobrev D (2013) Electrical storm: recent pathophysiological insights and therapeutic consequences. Basic Res Cardiol 108:336. doi:10.1007/s00395-013-0336-2

22. Yang T, Chun YW, Stroud DM, Mosley JD, Knollmann BC, Hong CC, Roden DM (2014) Screening for acute $I_{\mathrm{Kr}}$ block is insufficient to detect Torsades de pointes liability: role of Late sodium current. Circulation. doi:10.1161/CIRCULATIONAHA. 113.007765

23. Yokoshiki H, Katsube Y, Sunagawa M, Sperelakis N (1997) The novel calcium sensitizer levosimendan activates the ATP-sensitive $\mathrm{K}^{+}$channel in rat ventricular cells. J Pharmacol Exp Ther 283:375-383 